\title{
NuSTAR observation of GRO J1744-28 at low mass accretion rate
}

\author{
Ole König ${ }^{1}$, Felix Fürst ${ }^{2}$, Peter Kretschmar ${ }^{2}$, Ralf Ballhausen ${ }^{1}$, Ekaterina Sokolova-Lapa ${ }^{1,3}$, Thomas Dauser ${ }^{1}$, \\ Celia Sánchez-Fernández ${ }^{2}$, Paul B. Hemphill ${ }^{4}$, Michael T. Wolff ${ }^{5}$, Katja Pottschmidt ${ }^{6,7}$, and Jörn Wilms ${ }^{1}$ \\ 1 Dr. Karl-Remeis-Sternwarte and ECAP, Sternwartstr. 7, 96049 Bamberg, Germany \\ e-mail: ole.koenig@fau.de \\ 2 European Space Astronomy Centre (ESAC), Camino Bajo del Castillo, s/n, Urb. Villafranca del Castillo, 28692 Villanueva de la \\ Cañada, Madrid, Spain \\ 3 Sternberg Astronomical Institute, M. V. Lomonosov Moscow State University, Universitetskij Pr., 13, Moscow 119992, Russia \\ ${ }^{4}$ MIT Kavli Institute for Astrophysics and Space Research, 77 Massachusetts Ave., Cambridge, MA 02139, USA \\ 5 Space Science Division, Naval Research Laboratory, Washington DC 20375, USA \\ 6 CRESST, Department of Physics, and Center for Space Science and Technology, University of Maryland Baltimore County, 1000 \\ Hilltop Circle, Baltimore, MD 21250, USA \\ 7 NASA Goddard Space Flight Center, Code 661, 8800 Greenbelt Road, Greenbelt, MD 20771, USA
}

Received 29 July 2020 / Accepted 11 September 2020

\begin{abstract}
Context. Neutron stars in low-mass X-ray binaries are important systems for studying the physics of accretion onto compact objects. The system GRO J1744-28 is particularly interesting as it usually shows clear pulsations as well as X-ray bursts. Additionally, there are claims for a magnetic field of $5 \times 10^{11} \mathrm{G}$ through the detection of a cyclotron resonant scattering feature (CRSF).

Aims. We present the spectral analysis of GRO J1744-28 using $29 \mathrm{ks}$ of NuSTAR data taken in 2017 February at a low luminosity of $3.2 \times 10^{36} \mathrm{erg} \mathrm{s}^{-1}(3-50 \mathrm{keV})$. Our goal is to study the variability of the source spectrum with pulse phase and to search for the claimed CRSF.

Methods. The continuum spectrum was modeled with an absorbed power law with exponential cutoff, and an additional iron line component. We found no obvious indications for a CRSF, and therefore performed a detailed cyclotron line search using statistical methods. We performed this search on pulse phase-averaged spectra and on phase-resolved spectra.

Results. GRO J1744-28 was observed in a low-luminosity state. The previously detected Type II X-ray bursts are absent. Clear pulsations at a period of $2.141124(9) \mathrm{Hz}$ are detected. The pulse profile shows an indication of a secondary peak that was not seen at higher flux. The upper limit for the strength of a CRSF in the $3-20 \mathrm{keV}$ band is $0.07 \mathrm{keV}$ (90\% CL), lower than the strength of the line found at higher luminosity.

Conclusions. The detection of pulsations shows that the source did not enter the "propeller" regime, even though the source flux of $4.15 \times 10^{-10} \mathrm{erg} \mathrm{cm}^{-2} \mathrm{~s}^{-1}$ was almost one order of magnitude below the threshold for the propeller regime claimed in previous studies on this source. The transition into the propeller regime in GRO J1744-28 must therefore be below a luminosity of $3.2 \times 10^{36} \mathrm{erg} \mathrm{s}^{-1}$ (3-50 keV), which implies a surface magnetic field $\lesssim 2.9 \times 10^{11} \mathrm{G}$ and mass accretion rate $\lesssim 1.7 \times 10^{16} \mathrm{~g} \mathrm{~s}^{-1}$. A change of the CRSF depth as function of luminosity is not unexpected and has been observed in other sources. This result possibly implies a change in emission geometry as function of mass accretion rate to reduce the depth of the line below our detection limit.
\end{abstract}

Key words. pulsars: individual: GRO J1744-28 - X-rays: binaries - stars: neutron - accretion, accretion disks - magnetic fields

\section{Introduction}

Accretion-powered X-ray pulsars are binary systems consisting of a neutron star and an optical companion. They are often classified by the mass and spectral type of the donor star. High-mass X-ray binaries (HMXBs; e.g., Chaty 2011; Paul \& Naik 2011; Reig 2011, and references therein) have donor stars of O/B type. The accretion is typically wind-fed or, in the case of $\mathrm{Be} / \mathrm{X}$-ray binaries, from the Be star's decretion disk, where the neutron star exhibits a magnetic field strength of $\sim 10^{12}-\sim 10^{13} \mathrm{G}$. Low-mass X-ray binaries (LMXBs, e.g., Bhattacharyya 2010), on the other hand, consist of a late-type donor star and accreting neutron star, typically with lower magnetic field strength $O\left(10^{8}-10^{9} \mathrm{G}\right)$. These systems are believed to be much older and have an accretion disk usually fed by Roche lobe overflow.

Some pulsars exhibit spectral features that allow a direct estimate of the neutron star's magnetic field. When ionized matter approaches the Alfvén radius (Alfvén 1968), it couples to the $B$-field lines of the neutron star. The plasma is then funneled to the poles, where it gets decelerated and forms accretion columns. Moreover, in the presence of a magnetic field the electrons' motion is quantized perpendicular to the field onto discrete energy states, the Landau levels (Landau \& Lifshitz 1965; Langer 1981). Inverse Compton scattering, which is mainly responsible for the hard X-ray radiation, becomes a resonant process in this case. The transition of the electrons between different Landau levels gives rise to absorption line-like features in the spectrum. These are referred to as cyclotron resonant scattering features (CRSFs). The CRSF energy allows us to infer the $B$-field strength at the line forming region via the " $12-B-12$ " rule (Canuto \& Ventura 1977),

$B \sim(1+z)\left(E_{\mathrm{c}} / 11.6 \mathrm{keV}\right) \times 10^{12} \mathrm{G}$,

where $z$ is the surface gravitational red shift. CRSFs are usually detected in HMXBs at $10-90 \mathrm{keV}$ due to their intrinsic high $B$-field strength. LMXBs typically do not exhibit these 
lines although there are some famous exceptions (e.g., Her X-1 Trümper et al. 1978). A comprehensive review of CRSF sources and their observation has been recently presented by Staubert et al. (2019), while Schwarm et al. (2017a,b) summarize their theoretical modeling.

GRO J1744-28 is a transient LMXB discovered on 1995 December 2 with the Burst And Transient Source Experiment (BATSE) on board the Compton Gamma Ray Observatory (Fishman et al. 1995; Kouveliotou et al. 1996). It is associated with a position near the Galactic center, at a distance of 7.5-8.5 kpc (Augusteijn et al. 1997; Nishiuchi et al. 1999), although smaller values have been reported as well (Sanna et al. 2017). The companion star is of Type G4 III (Gosling et al. 2007; Masetti et al. 2014), and has an inferred mass of $<0.3 M_{\odot}$ with inclination $i>$ $15^{\circ}$. Sanna et al. (2017) give the most recent orbital ephemeris, with the orbital period $P_{\text {orb }}=11.8358(5)$ days, the projected semi-major axis $a_{x} \sin (i)=2.639$ (1) light-sec, and the eccentricity $\epsilon<6 \times 10^{-3}$. The source has a magnetic field that is strong enough that X-ray pulsations at $2.14 \mathrm{~Hz}$ are observed (Finger et al. 1996), even though Type II X-ray bursts are also seen (Lewin et al. 1976). GRO J1744-28 has therefore been dubbed the Bursting Pulsar (Strickman et al. 1996). Pure thermonuclear Type I X-ray bursts have not been detected, which is consistent with the picture of a high magnetic field (Bildsten \& Brown 1997; Court et al. 2018). Cui (1997) reports that the pulsations cease at a flux limit of $2.3 \times 10^{-9} \mathrm{erg} \mathrm{cm}^{-2} \mathrm{~s}^{-1}$ and interprets this threshold as a "centrifugal barrier" where the magnetosphere halts the accretion flow. This is also known as the "propeller" effect. By equaling the co-rotation radius and the radius of the magnetosphere (e.g., Fürst et al. 2017), we can estimate the surface magnetic field from the luminosity threshold

$L_{\text {prop }} \simeq \frac{G M \dot{M}}{R} \simeq 7.3 \times 10^{37} k^{7 / 2} B_{12}^{2} P^{-7 / 3} M_{1.4}^{-2 / 3} R_{6}^{5} \mathrm{erg} \mathrm{s}^{-1}$,

where $k=0.5$ in the case of disk accretion (Ghosh \& Lamb 1978), $B$ is the magnetic field in units of $10^{12} \mathrm{G}, P$ is the rotational period of the neutron star, $M_{1.4}$ is its mass in units of $1.4 M_{\odot}$, and $R_{6}$ is its radius in units of $10^{6} \mathrm{~cm}$.

About one year after the 1995 outburst during which GRO J1744-28 was discovered, it underwent a similar outburst (1996 December; Woods et al. 1999; Doroshenko et al. 2015), followed by 18 years of quiescence. In early 2014, GRO J174428 went into outburst again, which triggered NuSTAR and Chandra (Younes et al. 2015) as well as XMM-Newton and INTEGRAL (D'Aì et al. 2015) observations. With $1.9 \times$ $10^{38} \mathrm{erg} \mathrm{s}^{-1}$ the peak luminosity of the 2014 outburst was extremely high for a typical X-ray binary, although still similar to the two earlier outbursts.

This paper focuses on the fourth activity period of GRO J1744-28 in 2017 February. The Swift/BAT (Krimm et al. 2013) monitoring light curve is shown in Fig. 1; the overall luminosity of the source was significantly less than that seen in previous outbursts. The pointed NUSTAR observations during this outburst were performed at a flux of $4.15 \times 10^{-10} \mathrm{erg} \mathrm{cm}^{-2} \mathrm{~s}^{-1}$, which gives the first opportunity to study GRO J1744-28 in a regime of low mass accretion rate.

Using XMM-Newton and INTEGRAL data collected during the 2014 outburst, D'Aì et al. (2015) reported a fundamental CRSF at $4.68 \pm 0.05 \mathrm{keV}$, with an indication of second and third harmonics at $10.4 \pm 0.1 \mathrm{keV}$ and $15.8_{-0.7}^{+1.3} \mathrm{keV}$. Shortly afterwards, Doroshenko et al. (2015) claimed evidence for a CRSF at $\sim 4.5 \mathrm{keV}$ in archival BeppoSAX data taken during the 1997 outburst. These claims make GROJ1744-28 one of the few LMXBs where a CRSF has been reported, and one of the very

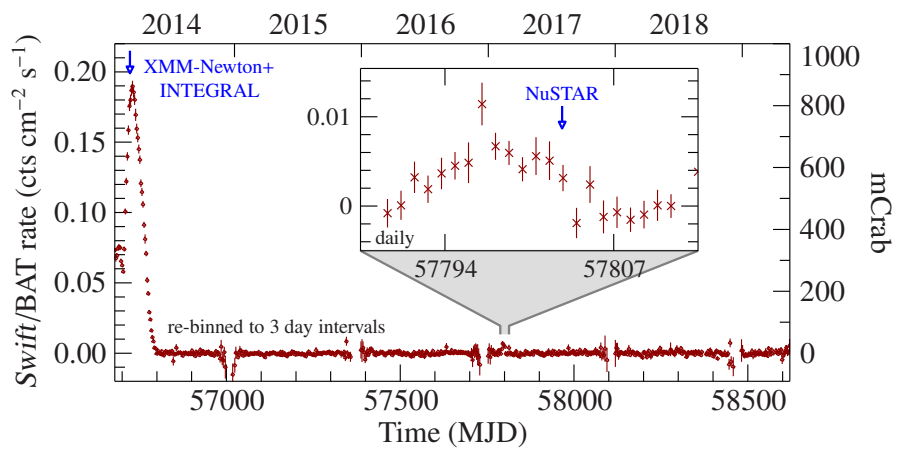

Fig. 1. Swift/BAT light curve of GRO J1744-28. The outburst in 2014 had a peak luminosity of $2.1 \times 10^{38} \mathrm{erg} \mathrm{s}^{-1}$, slightly above the Eddington limit (D'Aì et al. 2015). The outburst in 2017 is at much lower luminosity $3.2 \times 10^{36} \mathrm{erg} \mathrm{s}^{-1}(3-50 \mathrm{keV})$. The gaps in the Swift/BAT light curve are due to visibility constraints.

few sources with a reported CRSF energy below $10 \mathrm{keV}$. Other pulsars with low CRSFs are 4U 1822-371 with a claimed energy of $0.7 \mathrm{keV}$ (Iaria et al. 2015) and Swift J0051.8-7320 at $5 \mathrm{keV}$ (Maitra et al. 2018).

The detection of the CRSF in GRO J1744-28, however, is debated. Younes et al. (2015) did not find a significant CRSF in their data, which were taken only three days earlier than the D'Aì et al. (2015) detection. The polar magnetic field deduced from the CRSF energy, $5 \times 10^{11} \mathrm{G}$, is higher than those derived with different methods, for example $2-6 \times 10^{10} \mathrm{G}$ from accretion disk reflection modeling (Degenaar et al. 2014), $9 \times 10^{10} \mathrm{G}$ from a spin-up estimate (Younes et al. 2015), and $2.4 \times 10^{11} \mathrm{G}$ from the propeller effect flux threshold (Cui 1997).

In this paper we discuss our analysis of NUSTAR observations of GRO J1744-28 taken during its most recent outburst in 2017. In Sect. 2 we discuss the data extraction and calibration. We show that pulsations are clearly present in the data and present the results of the phase-averaged and phase-resolved spectroscopy in Sects. 3.1 and 3.2, respectively, and discuss the search for a CRSF. We discuss and summarize our results in Sect. 4.

\section{Data extraction and calibration}

The Nuclear Spectroscopic Telescope Array (NuSTAR; Harrison et al. 2013) has an energy range from $3-79 \mathrm{keV}$ and temporal resolution of $2 \mu \mathrm{s}$ which allows phase-resolved spectroscopy of rapidly rotating neutron stars. It has a moderate energy resolution of $400 \mathrm{eV}$ (FWHM) at $10 \mathrm{keV}$.

The NUSTAR data analyzed here have a net exposure of $28.8 \mathrm{ks}$ (FPM A) and 28.9 ks (FPM B) starting on 2017-02-18 14:34:35 UTC (MJD 57802.6073, ObsID: 80202027002), during the decay of the outburst. After standard cleaning for Earth occultation and the South Atlantic Anomaly according to the NUSTAR data analysis software guide ${ }^{1}$, we reduce the data with HEASOFT version 6.26 (corresponding to NuSTARDAS 1.8.0), using NUSTAR CalDB version 20190513. We barycenter the data and extract the source light curve and spectra from a circle of $60^{\prime \prime}$ radius centered on the source position. We do an orbit correction with the latest orbital parameters from Sanna et al. (2017), although we note that the orbit is still poorly constrained and the correction does not change the determined spin period

\footnotetext{
1 https://heasarc.gsfc.nasa.gov/docs/nustar/analysis/ nustar_swguide.pdf
} 
significantly. For the background extraction we define three circular regions of $120^{\prime \prime}$ radius for FPM B and two circles of the same size for FPM A (due to stray light contamination). We then average the counts per FPM A/B and scale them to the source area to increase the background statistics. The event files used to extract the phase-resolved spectra were filtered on the source region with XSELECT version $2.4 \mathrm{~g}$.

All further analysis was performed with the Interactive Spectral Interpretation System (ISIS version 1.6.2-43, Houck 2002). Unless stated otherwise, all error bars are at the $90 \%$ level single-parameter confidence level $\left(\Delta \chi^{2}=2.71\right)$. We restrict the data to the 3-78 keV range (PI channel 35-1210). We use the following binning scheme for the phase-averaged and phaseresolved spectra to account for NUSTAR's energy dependent energy resolution and oversample it by roughly a factor of 3 . In the energy range $3-10 \mathrm{keV}$ we group a minimum number of 2 channels per bin; in $10-15 \mathrm{keV}$ we group 3 channels, in $15-20 \mathrm{keV}$ we group 5; in 20-35 keV we group 8; in $35-45 \mathrm{keV}$ we group 16; in $45-55 \mathrm{keV}$ we group 18; in $55-65 \mathrm{keV}$ we group 48; in 65-76 keV we group 72; and >76 keV, we group 48 channels per bin, while also ensuring a minimum signal-to-noise ratio $(\mathrm{S} / \mathrm{N})$ of 5 .

\section{Spectral and timing analysis of GRO J1744-28}

\subsection{Phase-averaged spectrum}

In order to allow us to compare the continuum shape with earlier analyses, we use phenomenological continuum models rather than more physically motivated models such as those by Becker \& Wolff $(2007)^{2}$ or Farinelli et al. (2016). As discussed by Müller et al. (2013), among others, phenomenological spectral models typically used to describe the continua of accreting neutron stars are the exponentially cutoff power law (cutoffpl), the power law with Fermi-Dirac cutoff (FDcut, Tanaka 1986), a negative-positive cutoff power law (NPEX, Mihara 1995), and a model consisting of a blackbody disk (diskbb, Mitsuda et al. 1984) and thermally comptonized continuum (nthcomp, Zdziarski et al. 1996; Życki et al. 1999). The residuals of the cutoffpl, FDcut, NPEX, and diskbb+nthcomp models are shown in Fig. 2, and the best-fit parameters are given in Table 1. The NPEX and FDcut residuals look very similar because they are driven to parameters that effectively mimic the cutoffpl solution. All tested continuum models describe the data similarly well. Due to its simplicity and in order to allow comparison with previous work (e.g., Younes et al. 2015), we used the cutoffpl model for all subsequent analysis. Photoelectric absorption in the interstellar medium is accounted for with the tbnew model (TBabs in XSPEC) with cross sections and abundances according to Verner et al. (1996) and Wilms et al. (2000), respectively. The iron fluorescence line complex can formally be described by a slightly broadened $\left(\sigma=0.23_{-0.04}^{+0.05} \mathrm{keV}\right)$ Gaussian component at $6.59 \pm 0.04 \mathrm{keV}$. This is most likely a blend of different ionization states that cannot be resolved with NuSTAR. The strongest fluorescence lines are often produced by neutral $(6.4 \mathrm{keV})$, He-like $(6.7 \mathrm{keV})$, and H-like iron $(7.0 \mathrm{keV})$, and the structure seen in the data is also consistent with a set of narrow $\mathrm{K} \alpha$ lines from these ions, as well as neutral $\mathrm{K} \beta(7.1 \mathrm{keV})$ with a $\mathrm{K} \beta / \mathrm{K} \alpha$ flux ratio of $13 \%$ (Palmeri et al. 2003). With fixed energies and widths, this approach is also statistically valid and has the same degrees

\footnotetext{
2 The Becker \& Wolff (2007) model is also not applicable because the luminosity is too low to assume the presence of a radiation-dominated radiative shock.
}

of freedom as using one broad emission feature, but shows less interference with the continuum modeling because all line energies and widths are fixed and broadening is only due to the detector response. Using both approaches, slight residuals still remain at the iron $\mathrm{K}$ edge. These residuals are due to a combination of a gain-shift in NUSTAR energy calibration and the fact that the tbnew model only includes neutral iron.

The full model used for the X-ray continuum in our spectral fits with ISIS is therefore

$N_{\mathrm{ph}}(E)=$ tbnew $*$ const $*($ cutoffpl + Fe-complex $)$,

where the iron complex is modeled with Gaussian emission lines and the constant accounts for potential flux calibration uncertainties between FPM A and FPM B. This model gives a good description of the overall continuum shape $\left(\chi^{2} /\right.$ d.o.f. $=$ 462.2/407 = 1.14). The fit statistics of the best-fit FDcut, NPEX, and diskbb+nthcomp models are shown in Table 1. The observed 3-50 keV flux of $4.15 \times 10^{-10} \mathrm{erg} \mathrm{cm}^{-2} \mathrm{~s}^{-1}$ translates to a luminosity of $3.2 \times 10^{36} \mathrm{erg} \mathrm{s}^{-1}(3-50 \mathrm{keV})$, assuming spherical emission and a distance of $8 \mathrm{kpc}$. This is roughly two orders of magnitude lower than in the 1997 and 2014 outbursts, but three orders of magnitude brighter than the quiescent detections discussed by Daigne et al. (2002) and Wijnands \& Wang (2002) who see a softer spectrum.

\subsection{Phase-resolved spectra}

\subsubsection{Pulse period}

As the neutron star rotates, the line of sight onto the accretion column changes. Most X-ray pulsars therefore show spectral variations as a function of phase (see, e.g., Ferrigno et al. 2011). To extract phase-resolved spectra we first identify the local pulse period and define phase bins according to the hardness ratio, as described below. Using the epoch folding technique (Leahy et al. 1983; Schwarzenberg-Czerny 1989) we find a pulse period of $0.4670444(20) \mathrm{s}$, corresponding to a rotational frequency of $2.141124(9) \mathrm{Hz}$. The uncertainty is conservatively estimated by $\Delta P=P^{2} /\left(2 T_{\text {elapse }}\right)$. The determined pulse period is consistent with earlier measurements (Doroshenko et al. 2015; Younes et al. 2015; D'Aì et al. 2015), indicating that little spinup of the neutron star has happened since the 2014 outburst.

\subsubsection{Pulse profile and continuum parameters}

The top panel of Fig. 3 shows the pulse profile obtained by folding the energy-resolved light curves with the local pulse period. The profile was cleaned for the good time intervals (GTIs) of the observation and background subtracted. It shows one prominent peak spanning $\sim 1 / 3$ of the rotation, and a decrease in flux to a plateau at late phases where a secondary peak seems to be present around phase 0.75 . The colored histograms show the pulse profile in different energy bands. There are only subtle changes in the pulse spectral shape; a KS-test (Kolmogorov 1933; Smirnov 1939) yields no significant energy dependence. The chances for belonging to the same probability distribution is $96 \%$ when comparing the $3-8 \mathrm{keV}$ and $8-20 \mathrm{keV}$ pulse profiles, $59 \%$ for $3-8 \mathrm{keV}$ versus $20-78 \mathrm{keV}$, and $81 \%$ for $8-20 \mathrm{keV}$ versus $20-78 \mathrm{keV}$.

To study the spectral variability further, we calculate the hardness ratios as $(h-s) /(h+s)$, where $h$ is the count rate in the hard band, and $s$ the count rate in the soft band (Lightman \& Rybicki 1979). To define bins for the phaseresolved analysis we take the ratio of $3-5 \mathrm{keV}$ to $8-20 \mathrm{keV}$ : 


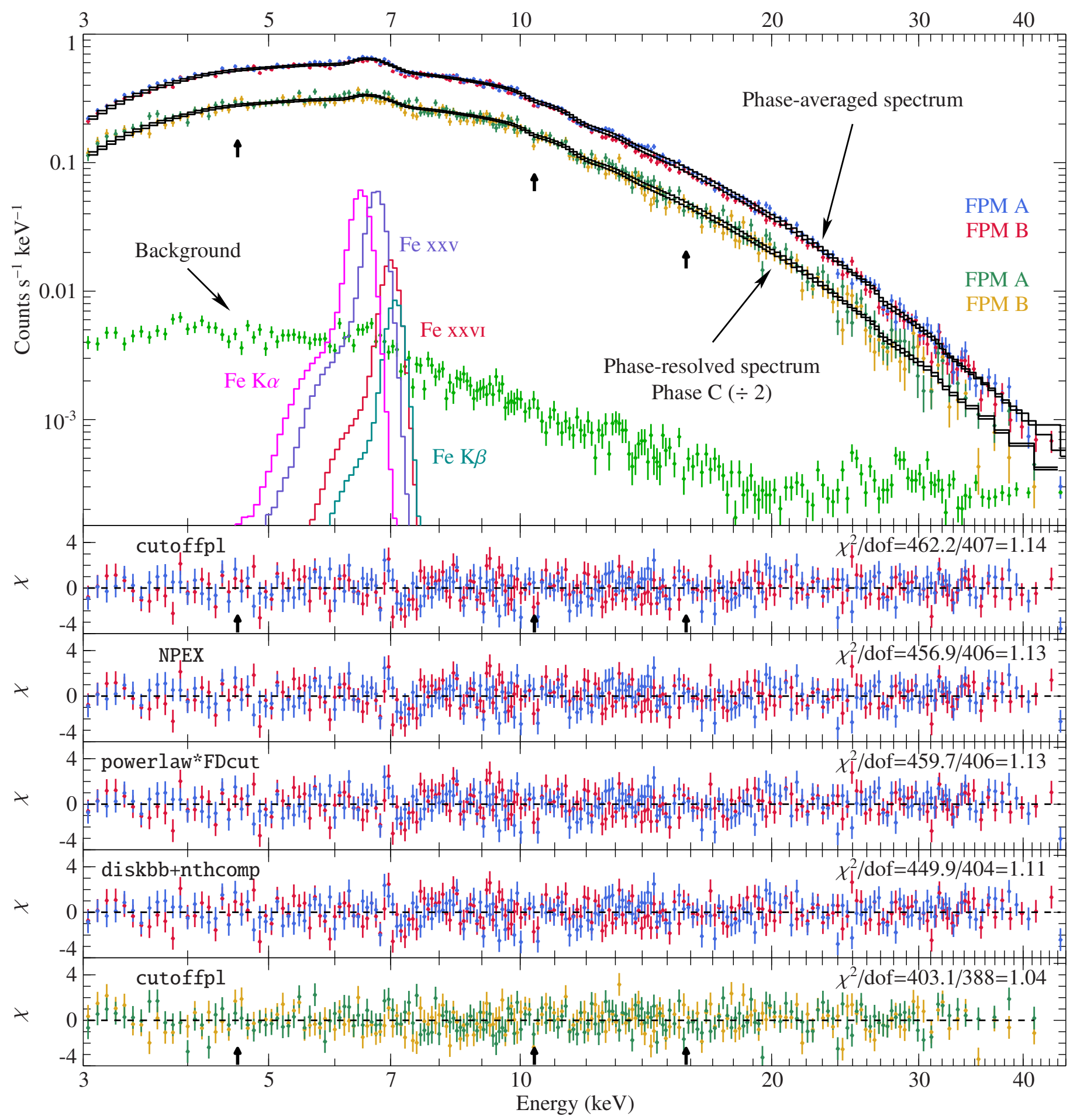

Fig. 2. Phase-averaged spectrum and one phase-resolved spectrum (phase $\mathrm{C}$, as defined in Fig. 3, displaced by factor of 2 for visualization). The black histogram gives the best-fitting model: an absorbed cutoff power law with iron component at $\sim 6.5 \mathrm{keV}$. Green points show background of FPM A. Arrows indicate the location of the reported CRSFs (Doroshenko et al. 2015; D'Aì et al. 2015). The iron line asymmetry is due to the convolution with the detector response and the logarithmic scale. All tested continuum models describe the data comparably well.

the hardness ratio of the soft band to the hard band. These energy bands provides a good compromise between energy resolution and $\mathrm{S} / \mathrm{N}$. To study the slight variations present in the hardness, we define six phase bins (A-F) of variable length to cover periods of largely constant hardness ratio. Since the different continuum parameters yield similar description of the phaseaveraged continuum, we model the phase-resolved data with the cutoffpl model only. No large changes as a function of phase are seen (Fig. 4), as expected from the near constant hardness ratio.

Finally, we turn to the amplitude of the pulsation, as shown in Fig. 5, as the energy dependent pulsed fraction, defined here as $\left(\mathrm{CR}_{\max }-\mathrm{CR}_{\min }\right) /\left(\mathrm{CR}_{\max }+\mathrm{CR}_{\min }\right)$, where $\mathrm{CR}$ denotes the count rate in the energy band's pulse profile. We emphasize that binning and the quality of the pulse profiles can have an effect on the measurement of the pulsed fraction, and that it is only a proxy 
Table 1. Fit parameters of phase-averaged spectrum for various models.

\begin{tabular}{|c|c|c|c|c|}
\hline Parameter & cutoffpl & NPEX & $\mathrm{pl} \times$ FDcut & diskbb + nthcomp \\
\hline$N_{\mathrm{H}}\left(10^{22} \mathrm{~cm}^{-2}\right)$ & $4.9 \pm 0.6$ & $4.5 \pm 0.6$ & $5.9 \pm 0.6$ & $10 \pm 4$ \\
\hline$\Gamma$ & $0.53 \pm 0.05$ & $0.25 \pm 0.04 ;-2^{(a)}$ & $0.83_{-0.04}^{+0.08}$ & $1.81_{-0.05}^{+0.06}$ \\
\hline$E_{\text {fold }}(\mathrm{keV})$ & $9.03_{-0.23}^{+0.24}$ & $5.56_{-0.20}^{+0.24}$ & $9.24 \pm 0.21$ & \\
\hline$E_{\text {cut }}(\mathrm{keV})$ & & & $\leq 3.19$ & \\
\hline Flux $\left(\times 10^{-10} \mathrm{erg} \mathrm{cm}^{-2} \mathrm{~s}^{-1}, 3-50 \mathrm{keV}\right)$ & $4.153 \pm 0.027$ & $4.139 \pm 0.026$ & $4.144 \pm 0.026$ & $4.140_{-0.027}^{+0.028}$ \\
\hline$k T_{\mathrm{e}}(\mathrm{keV})$ & & & & $5.99_{-0.22}^{+0.25}$ \\
\hline$k T_{\mathrm{BB}}(\mathrm{keV})$ & & & & $1.41_{-0.12}^{+0.20}$ \\
\hline$k T_{\text {disk }}(\mathrm{keV})$ & & & & $0.71_{-0.12}^{+0.29}$ \\
\hline Norm $_{\text {diskbb }}$ & & & & $30_{-40}^{+120}$ \\
\hline$E_{\mathrm{Fe} \mathrm{K} \alpha}(\mathrm{keV})^{(a),(c)}$ & 6.404 & 6.404 & 6.404 & 6.404 \\
\hline Flux $\left(10^{-4} \mathrm{ph} \mathrm{s}^{-1} \mathrm{~cm}^{-2}\right)$ & $1.23 \pm 0.26$ & $1.20 \pm 0.26$ & $1.25 \pm 0.26$ & $1.12_{-0.31}^{+0.30}$ \\
\hline$E_{\mathrm{Fe} \mathrm{K} \beta}(\mathrm{keV})^{(a),(c)}$ & 7.058 & 7.058 & 7.058 & $7.058^{-0.51}$ \\
\hline Flux $\left(10^{-5} \mathrm{ph} \mathrm{s}^{-1} \mathrm{~cm}^{-2}\right)^{(b)}$ & 1.6 & 1.5 & 1.6 & 1.4 \\
\hline$E_{\mathrm{Fexxv}}(\mathrm{keV})^{(a),(c)}$ & 6.7 & 6.7 & 6.7 & 6.7 \\
\hline Flux $\left(10^{-4} \mathrm{ph} \mathrm{s}^{-1} \mathrm{~cm}^{-2}\right)$ & $1.20 \pm 0.28$ & $1.20 \pm 0.28$ & $1.19 \pm 0.28$ & $1.11_{-0.30}^{+0.29}$ \\
\hline$E_{\mathrm{FexxvI}}(\mathrm{keV})^{(a),(c)}$ & 6.98 & 6.98 & 6.98 & $6.98^{-0.50}$ \\
\hline Flux $\left(10^{-5} \mathrm{ph} \mathrm{s}^{-1} \mathrm{~cm}^{-2}\right)$ & $3.3 \pm 2.3$ & $3.2 \pm 2.3$ & $3.8 \pm 2.3$ & $2.8_{-2.6}^{+2.5}$ \\
\hline$E_{\text {gabs }}(\mathrm{keV})$ & $7_{-4}^{+13}$ & & & \\
\hline$\sigma_{\text {gabs }}(\mathrm{keV})^{(a)}$ & 1.0 & & & \\
\hline Strength (keV) & $\leq 0.07$ & & & \\
\hline Normalization & $0.0141 \pm 0.0010$ & $0.0120_{-0.0009}^{+0.0010}\left(1.21_{-0.28}^{+0.27}\right) \times 10^{-3}$ & $0.0364_{-0.0037}^{+0.0025}$ & $\left(3.3_{-0.9}^{+0.8}\right) \times 10^{-3}$ \\
\hline Constant & $0.965 \pm 0.007$ & $0.965 \pm 0.007$ & $0.965 \pm 0.007$ & $0.965 \pm 0.007$ \\
\hline$\chi^{2}$ (d.o.f.) & $462.2 / 407$ & $456.9 / 406$ & $459.7 / 406$ & $449.9 / 404$ \\
\hline$\chi_{\text {red. }}^{2}$ & 1.14 & 1.13 & 1.13 & 1.11 \\
\hline
\end{tabular}

Notes. The underlying function for all fit models is tbnew $*$ const $*$ (continuum model $+\mathrm{Fe}$-complex) where the continuum model is specified in the column header. The upper limit on a cyclotron line is discussed in Sect. 3.1. Uncertainties are at the 90\% confidence level. We use wilm abundances and vern cross sections. ${ }^{(a)}$ Parameter frozen. ${ }^{(b)}$ Tied to $0.13 \cdot$ Flux $_{\mathrm{Fe} \alpha}$, see Palmeri et al. (2003). ${ }^{(c)}$ Narrow line with frozen width $\sigma=10^{-6} \mathrm{keV}$.

for assessing the pulsations. The pulsed fraction is $9.5 \pm 1.1 \%$ at energies $3-6 \mathrm{keV}$ and $12.9 \pm 2.8 \%$ above $9 \mathrm{keV}$, consistent with Younes et al. (2015). We see a tentative dip at $7 \mathrm{keV}$ where the iron line is located. This is consistent with the picture that this fluorescence line originates from outside of the accretion column or hot-spot. The pulsed fraction over the full $3-78 \mathrm{keV}$ range is $8.2 \pm 0.6$. We note that increases at high energies have also been seen in other X-ray pulsars (e.g., Lutovinov \& Tsygankov 2009).

\subsection{Search for cyclotron resonant scattering feature}

\subsubsection{Cyclotron line search in phase-averaged spectrum}

As discussed in Sect. 1, GRO J1744-28 is among the CRSF candidates with the lowest line energies proposed to date. A detailed search in this NUSTAR observation at a luminosity around two orders of magnitude lower than in previous outbursts is therefore of particular interest. Since the residuals of our best-fit model without a CRSF (Fig. 2) do not show absorption line-like residuals, the CRSF in the present observation must be weak or absent. We therefore perform a systematic search for a line in order to at least find limits for its parameters. The most common phenomenological model is the multiplicative Gaussian absorption line (gabs) of the form $\exp [-\tau(E)]$ with the Gaussian-shaped optical depth

$\tau(E)=\tau_{0} \exp \left[-\frac{\left(E-E_{\mathrm{c}}\right)^{2}}{2 \sigma^{2}}\right]$ and $\tau_{0}$ the central optical depth. This component introduces the parameter strength (in $\mathrm{keV}$ ) equaling $\tau_{0} \sqrt{2 \pi} \sigma$, which is widely used to determine the significance of cyclotron lines (e.g., Pottschmidt et al. 2005; Lutovinov et al. 2017). The width of the line, $\sigma$, is mainly constrained by the electron temperature and the viewing angle (e.g., Heindl et al. 2004; Schwarm et al. 2017b). Based on Meszaros \& Nagel (1985), Staubert et al. (2019) predict a width of $\sim 1 \mathrm{keV}$ at the reported $5 \mathrm{keV}$ energy. Doroshenko et al. (2015) found a width of $1.2 \pm 0.3 \mathrm{keV}$, whereas D'Aì et al. (2015) found a smaller width of $0.68 \pm 0.08 \mathrm{keV}$. The weakness of any line in the spectrum will make it impossible to constrain both its energy and width. Motivated by these earlier observations we therefore fix its width to $1 \mathrm{keV}$ and perform a systematic search by stepping through the $3-20 \mathrm{keV}$ band in 1000 steps while fitting for the line. This, however, does not give an improvement in $\chi^{2}$. The data yield an upper limit of $0.07 \mathrm{keV}$ ( $90 \%$ CL) on the strength of a gabs component. Attempts to model the spectrum with a CRSF with varying width did not result in physical values.

\subsubsection{Cyclotron line search in phase-resolved spectra}

Although no significant cyclotron line is found in the phaseaveraged spectrum, it might still be possible that the line is present in phase-resolved data. The reason is that CRSF produced in localized regions in the accretion column might only be visible during certain phases of the neutron star rotation. 


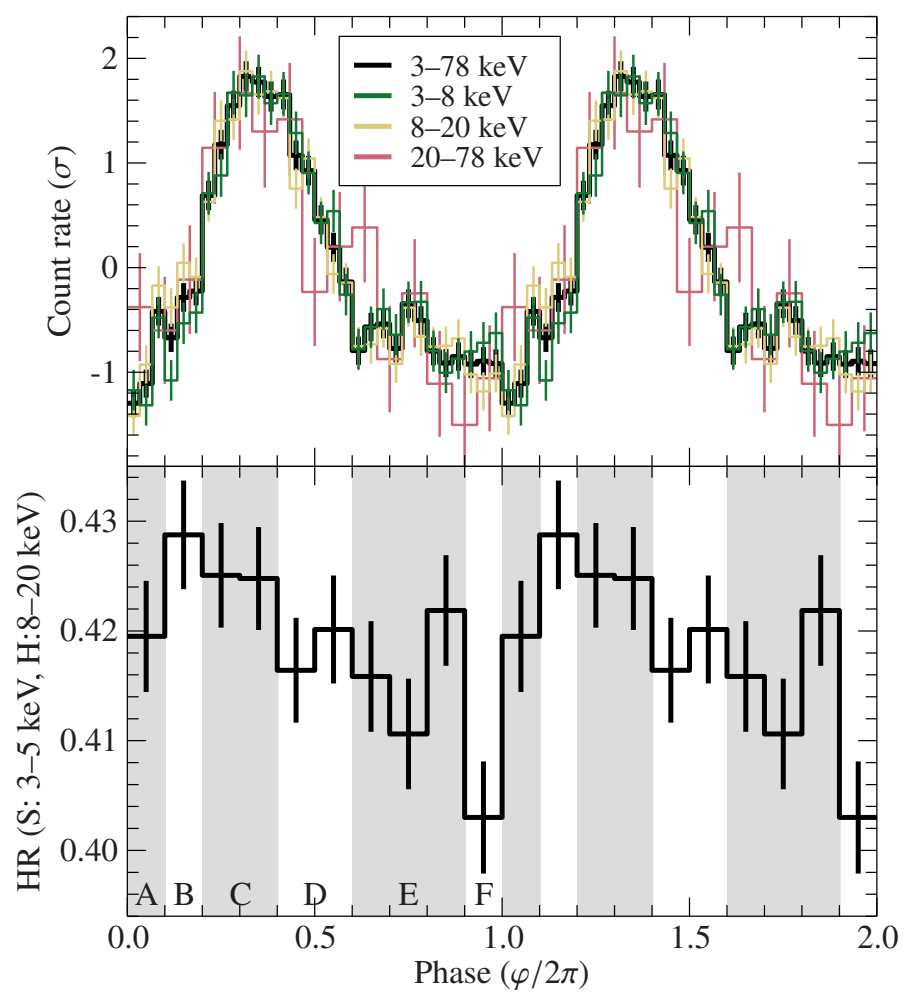

Fig. 3. Top: background-subtracted and GTI-corrected pulse profile of GRO J1744-28 for the 3-78 keV band (black) and for three narrower energy bands (colored). The count rate is normalized by subtracting the mean and dividing by the standard deviation to emphasize potential changes in the shape of the profile. The $20-78 \mathrm{keV}$ band is binned more coarsely for visualization purposes. No significant energy dependence can be seen (see text). Bottom: variation in the hardness ratio with phase (combined data from FPM A and B). The vertical bands show the phase ranges chosen for phase-resolved spectroscopy.

To search for such a line, similar to our phase-averaged spectral analysis, we include a Gaussian absorption component of fixed energy and width, and only fit the line strength. Based on the earlier data summarized in Sect. 1, we vary the centroid energy from $3 \mathrm{keV}$ to $20 \mathrm{keV}$ and determine the $\chi^{2}$ improvement at each sampled energy. We again fix the line width to $1 \mathrm{keV}$ (see also Staubert et al. 2019, Fig. 12). We repeat this procedure for all phase intervals and show the result in Fig. 6. Since one cannot use simple likelihood ratio tests for the presence of a line (Protassov et al. 2002), in order to see whether there are significant deviations from the model without absorption lines, we use the Akaike Information Criterion (Akaike 1974). For small sample sizes, this is computed by AIC $=\chi+2 k+\left(2 k^{2}+2 k\right) /(n-k-1)$ where $k$ is the number of free parameters and $n$ the number of bins ( $n-k$ is the number of degrees of freedom). We cannot find any significant deviation in the resulting $\Delta \chi^{2}$ distribution of Fig. 6: No phase exhibits a significance larger than $2 \sigma$. Specifically, the largest $\Delta \chi^{2}$ in phase $C$ yields a chance improvement probability, $\exp \left(-\Delta_{\mathrm{AIC}} / 2\right)$, of $20 \%$. It is therefore very likely that the slight increase in $\chi^{2}$ found when including a CRSF is only due to statistical effects.

\subsubsection{Monte Carlo simulations}

In Sect. 3.3.1, we determined an upper limit of $0.07 \mathrm{keV}$ on the strength of a CRSF. As the gabs strength of D'Aì et al. (2015) and Doroshenko et al. (2015) is above this value (with $0.087 \mathrm{keV}$

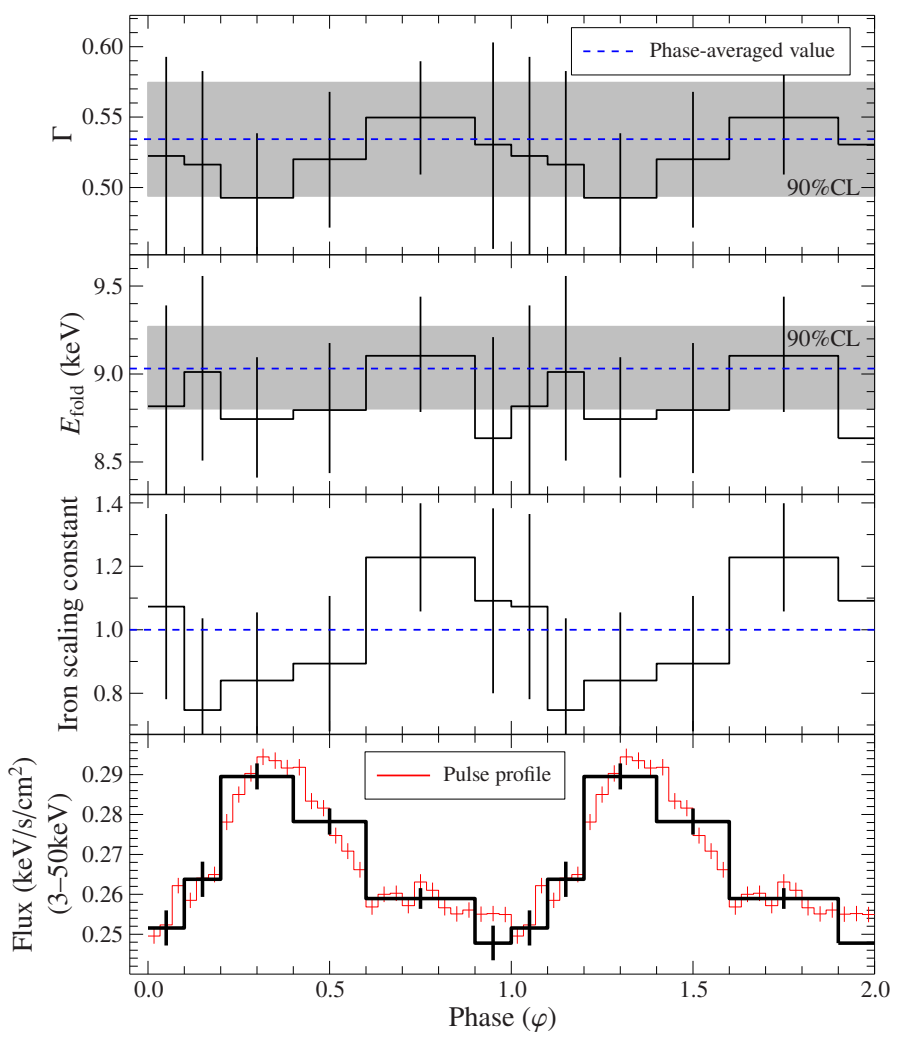

Fig. 4. Phase-resolved continuum parameters of the fit model. The blue dashed line gives the phase-averaged values at the $90 \%$ confidence level (shaded). The FPM A and FPM B cross-calibration constant 0.965 and the column density $6.35 \times 10^{22} \mathrm{~cm}^{-2}$ are fixed to the phase-averaged best-fit values. The "iron scaling constant" shows the variation in the relative strength of the iron line complex, whose parameters have been otherwise fixed to the phase-averaged values. No significant changes can be identified in the continuum parameters. Lowest panel: we plot the (re-scaled) pulse profile. Errors are at the $90 \%$ confidence level.

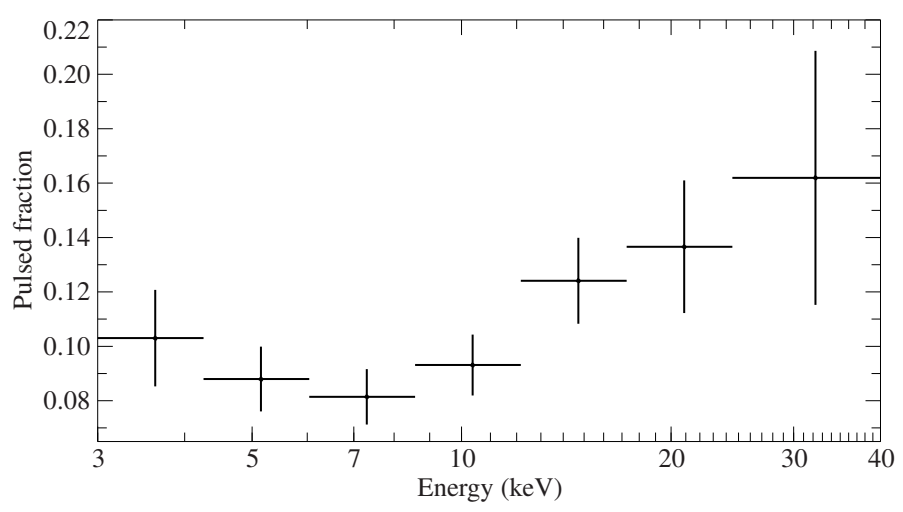

Fig. 5. Pulsed fraction $\left(\mathrm{CR}_{\max }-\mathrm{CR}_{\min }\right) /\left(\mathrm{CR}_{\max }+\mathrm{CR}_{\min }\right)$. The dip at $7 \mathrm{keV}$ is most likely due to the iron fluorescence line. The increase at higher energies is consistent with previous findings.

and $\sim 0.12 \mathrm{keV}$, respectively), we can rule out a line as strong as previously claimed at the $90 \%$ level. We therefore would likely have seen a trace of the CRSF (with $\sim 2-3 \sigma$ ) if it was as strong as previously reported. This is illustrated in Fig. 7 where we plot the CRSF properties of previous claims into the residuals of our NuSTAR data.

In this section we discuss how strong the cyclotron line would have to be in order to be significantly detected in our NUSTAR data. We simulate 20000 fake spectra based on the 


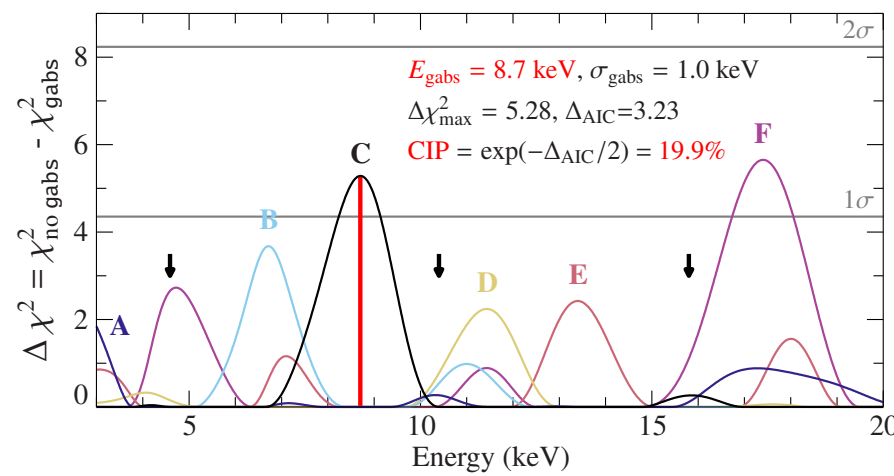

Fig. 6. $\Delta \chi^{2}$ for inclusion of a CRSF plotted as a function of energy for all phase-resolved spectra. No phase shows an absorption feature with $>2 \sigma$ significance. Black arrows show the reported CRSF energies (Doroshenko et al. 2015; D’Aì et al. 2015).

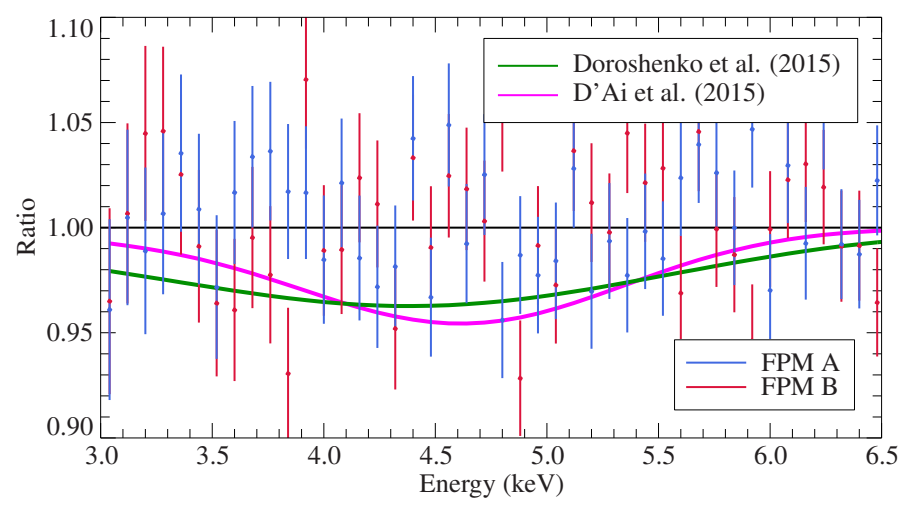

Fig. 7. Ratio of NUSTAR data and fit model without gabs as a function of energy in the band where a CRSF was observed in earlier data. The green and pink lines show the CRSF parameters claimed in earlier analyses. CRSFs with these strengths would have likely been seen in the present data data.

exposure and best-fit model of the phase-averaged spectrum (without gabs). The data points are drawn from a Poisson distribution with mean at the model value. The $\mathrm{S} / \mathrm{N}$ of the data directly affects the number of absorption features emerging due to statistical fluctuations. By analyzing the number of spurious detections of lines we put a lower limit on the cyclotron line strength and determine whether we would have been able to see the CRSF as previously reported.

We fit the simulated spectrum with the best phase-averaged fit model plus an additional Gaussian absorption feature (gabs) and extract its strength and energy (Fig. 8). We constrain the fitted line energy to be above $4 \mathrm{keV}$ in order to avoid the line running into NuSTAR's lower energy limit. The starting value of the line energy is $5 \mathrm{keV}$. Additionally, we freeze the width to $1 \mathrm{keV}$ as before; otherwise, the width is almost always fitted to the lowest possible value.

The Monte Carlo simulations of Fig. 8 show that many spurious lines are above the values of previous reports. For a robust detection claim, we would like to detect a line with a $4 \sigma$ confidence level. In order to determine how strong such a cyclotron line must be in our NUSTAR data, we scan the gabs strength distribution for the value where it exceeds the required false rate of $6.3 \times 10^{-5}(4 \sigma)$. Thus, we put a $4 \sigma$ detection limit of $0.15 \mathrm{keV}$ on the strength of the cyclotron line.

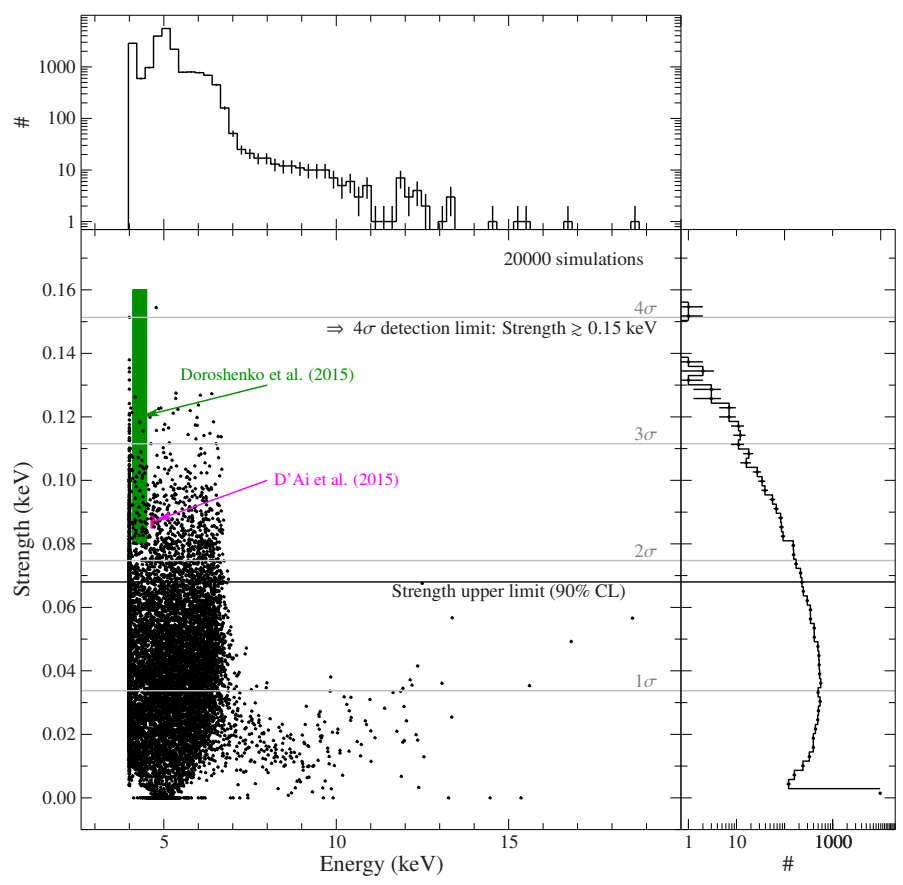

Fig. 8. Distribution of energy and strength of the best-fit CRSF found in a Monte Carlo simulation of 20000 fake spectra that do not include a CRSF. Statistical fluctuations will lead to artificial absorption features, fitted with a gabs model. We determine a $4 \sigma$ detection limit of $0.15 \mathrm{keV}$ on the strength of a significant CRSF detection. $7 \%$ of the fits are above the phase-averaged $90 \%$ CL upper limit strength of $0.07 \mathrm{keV}$.

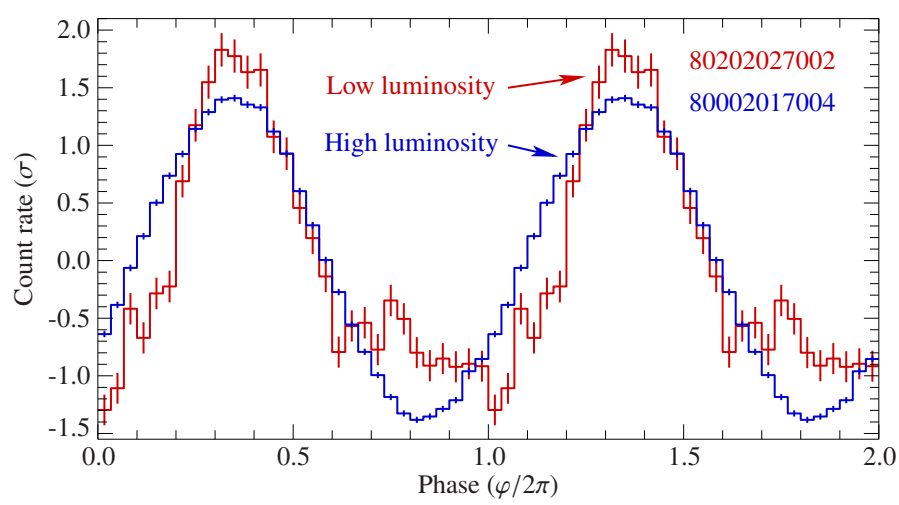

Fig. 9. NUSTAR pulse profiles of this observation (red) taken at a luminosity of $3.2 \times 10^{36} \mathrm{erg} \mathrm{s}^{-1}(3-50 \mathrm{keV})$ and the 2014 outburst (blue) at $1.9 \times 10^{38} \mathrm{erg} \mathrm{s}^{-1}(0.5-10 \mathrm{keV}$, Younes et al. 2015). The pulse profile of this observation is narrower, less sinusoidal and shows the indication of a secondary peak at late phases.

\section{Discussion and summary}

\subsection{Discussion}

In this paper we presented a spectral analysis of the fourth recorded outburst of the X-ray pulsar GRO J1744-28 in a lowflux state, which occurred in 2017 February. In contrast to previous outbursts, the source only reached a luminosity of $3.2 \times$ $10^{36} \mathrm{erg} \mathrm{s}^{-1}(3-50 \mathrm{keV})$, assuming a distance of $8 \mathrm{kpc}$. We note, however, that this is still three orders of magnitude above quiescence level (Daigne et al. 2002; Wijnands \& Wang 2002).

The spectral shape found during our low luminosity observation can be well described by an absorbed cutoff power law with an additional fluorescence iron emission line complex at 
$\sim 6.6 \mathrm{keV}$. The spectral shape is also consistent with a NPEX, FDcut, or nthcomp model, which were used in some of the earlier studies of GRO J1744-28. For this reason, a direct comparison of the model parameters is difficult. However, a comparison of the spectral shapes found in earlier data (Doroshenko et al. 2015; Younes et al. 2015; Cui 1998) shows a slightly softer spectral shape in this low luminosity observation. This softening with decreasing luminosity is consistent with previous studies of GROJ1744-28 (Cui 1998; Daigne et al. 2002; Wijnands \& Wang 2002), and generally on accreting pulsars (Reig \& Nespoli 2013; Postnov et al. 2015). We note that we do not see a double-humped structure in the spectrum as seen in a few accreting X-ray pulsars at even lower luminosity (Tsygankov et al. 2019a,b).

The most debated feature in the X-ray spectrum is the existence of the cyclotron line. CRSF are difficult to detect below $10 \mathrm{keV}$, due to other spectral features in this regime, and a confirmation of the $\sim 5 \mathrm{keV}$ line would make GRO J1744-28 one of the few secure neutron star systems with a weak magnetic field. The existing claims for CRSFs in GRO J1744-28 were at a luminosity $2.4 \times 10^{38} \mathrm{erg} \mathrm{s}^{-1}(d=8 \mathrm{kpc})$, slightly above its Eddington luminosity (D'Aì et al. 2015) and in the super-critical accretion regime (Becker et al. 2012). However, even at these high luminosities the CRSF was not always seen. Just three days before the detection of D'Aì et al. (2015), Younes et al. (2015) did not detect a CRSF, and while Doroshenko et al. (2015, their Table 3), observed the line in the brightest state of the 1997 outburst, it vanished $(\tau=0)$ at later times when the luminosity had decreased to $0.46 \times 10^{37} \mathrm{erg} \mathrm{s}^{-1}(2-10 \mathrm{keV})$.

During this NuSTAR observation GRO J1744-28 had a luminosity of $3.2 \times 10^{36} \mathrm{erg} \mathrm{s}^{-1}(3-50 \mathrm{keV})$, two orders of magnitude below the Eddington luminosity. Neither the phase-averaged nor the phase-resolved spectrum exhibit a significant cyclotron resonant scattering feature, with an upper limit for the strength of $0.07 \mathrm{keV}(90 \% \mathrm{CL})$. There are several possible reasons for this vanishing of the cyclotron line. First, in some sources we see that the line energy depends on luminosity (e.g., Staubert et al. 2019, and references therein). Therefore, it is possible that the line energy strongly depends on luminosity such that it could be located below the lower energy threshold of NuSTAR. Such a dependency, however, would require a large increase in the height of the accretion column, while we expect accretion columns at low luminosities to be small (e.g., Becker et al. 2012; Nishimura 2014; Mushtukov et al. 2015). Alternatively, the large luminosity difference could have changed the emission geometry such that the conditions at which the line is formed are not met. Finally, it could also be that the CRSF seen in earlier data is an artifact of the continuum modeling with simple empirical models.

Even though the source was at a flux of only $4.15 \times$ $10^{-10} \mathrm{erg} \mathrm{cm}^{-2} \mathrm{~s}^{-1}$, we still detect pulsations. The pulse profile shows a prominent peak, and a smaller secondary peak at phase $\sim 0.75$ is apparent that was not seen in the earlier higher luminosity data, which were smooth and almost perfectly sinusoidal (e.g., Doroshenko et al. 2015; D’Aì et al. 2015; Younes et al. 2015, and Fig. 9). The spectral shape is only very slightly variable with pulse phase.

Our observation of distinct pulsations (and thus the presence of a hot spot or an accretion column) at such a low flux contradicts earlier RXTE analyses, where the propeller effect was claimed to set in at a flux of $2.34 \times 10^{-9} \mathrm{erg} \mathrm{cm}^{-2} \mathrm{~s}^{-1}$ (Cui 1997). Even though RXTE had a much larger effective area than NUSTAR, the background level in the RXTE PCA was much higher. We therefore speculate that the non-detection of pulsations at higher flux was due to the lower $\mathrm{S} / \mathrm{N}$ of the earlier observations. With the newer NUSTAR data we can therefore revise the threshold for the transition into the propeller regime to below $4.15 \times 10^{-10} \mathrm{erg} \mathrm{cm}^{-2} \mathrm{~s}^{-1}(3-50 \mathrm{keV})$, i.e., to almost an order of magnitude below the value found earlier. If we assume that the resulting luminosity is the transitional threshold for the propeller regime we can constrain the surface magnetic field strength to $B \lesssim 2.9 \times 10^{11} \mathrm{G}$ (for a canonical neutron star, see Eq. (2)) and the mass accretion rate to $\dot{M} \lesssim 1.7 \times 10^{16} \mathrm{~g} \mathrm{~s}^{-1}$. The $B$-field estimate is in line with previous estimates on the source (Degenaar et al. 2014; Younes et al. 2015). We note that the value would imply a red-shifted CRSF at $\lesssim 2.6 \mathrm{keV}$, which is outside of NUSTAR's energy range and about $2 \mathrm{keV}$ lower than the claimed CRSF.

We caution that there are several different versions of the propeller luminosity (Eq. (2)) in use, depending on the underlying assumptions about accretion geometry and magnetic field configuration. For example Campana et al. (2001, Eq. (2) and evaluating their $B_{0}$ ) use a pre-factor of $1.69 \times$ $10^{37} \mathrm{erg} \mathrm{s}^{-1}$, Tsygankov et al. (2017, Eq. (4)) of $4 \times 10^{37} \mathrm{erg} \mathrm{s}^{-1}$, and Fürst et al. (2017, Eq. (2)) of $7.3 \times 10^{37} \mathrm{erg} \mathrm{s}^{-1}$. Furthermore, different authors use different values of the accretion geometry parameter $k$. This can result in relatively large differences in the $B$-field estimate, to be specific $B \propto\left(1 /\right.$ prefac $^{1 / 2} k^{-7 / 4}$. If we evaluate the equation by Cui $(1997, k=1$ and a pre-factor of $4.8 \times 10^{37} \mathrm{erg} \mathrm{s}^{-1}$ ), we obtain a $B$-field of $B \lesssim 1.0 \times 10^{11} \mathrm{G}$, which is a difference of factor 2.8 to our parameter choice.

Finally, we turn to the occurrence of X-ray bursts. Earlier observations at persistent fluxes ranging from $10^{-8}$ $10^{-9} \mathrm{erg} \mathrm{cm}^{-2} \mathrm{~s}^{-1}$ (Kouveliotou et al. 1996; Jahoda et al. 1996; Woods et al. 1999; Younes et al. 2015) showed the rate of $\mathrm{X}$-ray bursts to decrease with flux from $\sim 20 \mathrm{~h}^{-1}$ to $1 \mathrm{~h}^{-1}$ (Kouveliotou et al. 1996). In quiescence, no X-ray bursts were observed (Daigne et al. 2002; Wijnands \& Wang 2002), and neither did we see evidence of X-ray bursts here. This indicates that at a flux of $4.15 \times 10^{-10} \mathrm{erg} \mathrm{cm}^{-2} \mathrm{~s}^{-1}$, GRO J1744-28 must be in a regime where the burst rate is less than $\sim 0.1 \mathrm{~h}^{-1}$, possibly even implying that Type II X-ray bursts cease below a certain mass accretion rate.

In order to place further constraints on both the transition flux to the propeller regime and on the existence of a cyclotron line, further observations are necessary with better $\mathrm{S} / \mathrm{N}$, for instance with the Neutron Star Interior Composition Explorer (NICER).

\subsection{Summary}

Our most important results of this spectral analysis of the 2017 outburst with $N U S T A R$ are the following:

- GRO J1744-28 had a luminosity of $3.2 \times 10^{36} \mathrm{erg} \mathrm{s}^{-1}$ (3-50 keV), two orders of magnitude below previous outbursts but three orders of magnitude above quiescence level.

- The light curve shows no Type II X-ray bursts, which means that the burst rate is less than $\sim 0.1 \mathrm{~h}^{-1}$.

- The power-law spectrum is slightly softer than in the highluminosity case.

- We cannot find a significant CRSF in the spectrum, and put a $4 \sigma$ detection limit of $0.15 \mathrm{keV}$ on the gabs strength.

- The presence of pulsations allows us to set the threshold for the transition into the propeller regime to below $4.15 \times$ $10^{-10} \mathrm{erg} \mathrm{cm}^{-2} \mathrm{~s}^{-1}$, almost an order of magnitude lower than previously found. The resulting surface magnetic field can be constrained to $\lesssim 2.9 \times 10^{11} \mathrm{G}$ and the mass accretion rate to $\lesssim 1.7 \times 10^{16} \mathrm{~g} \mathrm{~s}^{-1}$. 
Acknowledgements. OK thanks the ESAC Trainee Program (https://www. cosmos.esa.int/web/esac-trainees) which initiated this analysis, and the ERASMUS+ fellowship program for their financial support. RB acknowledges funding by Deutsches Zentrum für Luft- und Raumfahrt under contract 50OR 1606. MTW is supported by the NUSTAR Guest Investigator Program. This research has made use of ISIS functions (ISISscripts) provided by ECAP/Remeis observatory and MIT (http://www.sternwarte. uni-erlangen.de/isis/). This research has made use of the NuSTAR Data Analysis Software (NuSTARDAS) jointly developed by the ASI Science Data Center (ASDC, Italy) and the California Institute of Technology (USA).

\section{References}

Akaike, H. 1974, IEEE Trans. Autom. Control, 19, 716

Alfvén, H. 1968, J. Geophys. Res., 73, 4379

Augusteijn, T., Greiner, J., Kouveliotou, C., et al. 1997, ApJ, 486, 1013

Becker, P. A., \& Wolff, M. T. 2007, ApJ, 654, 435

Becker, P. A., Klochkov, D., Schönherr, G., et al. 2012, A\&A, 544, A123

Bhattacharyya, S. 2010, Adv. Space Res., 45, 949

Bildsten, L., \& Brown, E. F. 1997, ApJ, 477, 897

Campana, S., Gastaldello, F., Stella, L., et al. 2001, ApJ, 561, 924

Canuto, V., \& Ventura, J. 1977, Fund. Cosm. Phys., 2, 203

Chaty, S. 2011, in Evolution of Compact Binaries, eds. L. Schmidtobreick, M. R. Schreiber, \& C. Tappert, ASP Conf. Ser., 447, 29

Court, J. M. C., Altamirano, D., Albayati, A. C., et al. 2018, MNRAS, 481, 2273 Cui, W. 1997, ApJ, 482, L163

Cui, W. 1998, in Accretion Processes in Astrophysical Systems, eds. S. S. Holt, \& T. R. Kallman, AIP Conf. Ser., 431, 405

D’Aì, A., Di Salvo, T., Iaria, R., et al. 2015, MNRAS, 449, 4288

Daigne, F., Goldoni, P., Ferrando, P., et al. 2002, A\&A, 386, 531

Degenaar, N., Miller, J. M., Harrison, F. A., et al. 2014, ApJ, 796, L9

Doroshenko, R., Santangelo, A., Doroshenko, V., et al. 2015, MNRAS, 452, 2490

Farinelli, R., Ferrigno, C., Bozzo, E., \& Becker, P. A. 2016, A\&A, 591, A29

Ferrigno, C., Falanga, M., Bozzo, E., et al. 2011, A\&A, 532, A76

Finger, M. H., Koh, D. T., Nelson, R. W., et al. 1996, Nature, 381, 291

Fishman, G. J., Kouveliotou, C., van Paradijs, J., et al. 1995, IAU Circ., 6272, 1

Fürst, F., Kretschmar, P., Kajava, J. J. E., et al. 2017, A\&A, 606, A89

Ghosh, P., \& Lamb, F. K. 1978, ApJ, 223, L83

Gosling, A. J., Bandyopadhyay, R. M., Miller-Jones, J. C. A., \& Farrell, S. A. 2007, MNRAS, 380, 1511

Harrison, F. A., Craig, W. W., Christensen, F. E., et al. 2013, ApJ, 770, 103

Heindl, W. A., Rothschild, R. E., Coburn, W., et al. 2004, in X-ray Timing 2003: Rossi and Beyond, eds. P. Kaaret, F. K. Lamb, \& J. H. Swank, AIP Conf. Ser. 714,323

Houck, J. C. 2002, in High Resolution X-ray Spectroscopy with XMM-Newton and Chandra, ed. G. Branduardi-Raymont (Dorking: Mullard Space Science Laboratory), 17

Iaria, R., Di Salvo, T., Matranga, M., et al. 2015, A\&A, 577, A63

Jahoda, K., Strohmayer, T., Corbet, R., \& Stark, M. 1996, IAU Circ., 6414, 2

Kolmogorov, A. N. 1933, Giornale dell'Instituto Italiano degli Attuari, 4 83
Kouveliotou, C., van Paradijs, J., Fishman, G. J., et al. 1996, Nature, 379, 799 Krimm, H. A., Holland, S. T., Corbet, R. H. D., et al. 2013, ApJS, 209, 14

Landau, L. D., \& Lifshitz, E. M. 1965, Quantum Mechanics (Oxford: Pergamon Press)

Langer, S. H. 1981, Phys. Rev. D, 23, 328

Leahy, D. A., Elsner, R. F., \& Weisskopf, M. C. 1983, ApJ, 272, 256

Lewin, W. H. G., Doty, J., Clark, G. W., et al. 1976, ApJ, 207, L95

Lightman, A. P., \& Rybicki, G. B. 1979, ApJ, 232, 882

Lutovinov, A. A., \& Tsygankov, S. S. 2009, Astron. Lett., 35, 433

Lutovinov, A. A., Tsygankov, S. S., Postnov, K. A., et al. 2017, MNRAS, 466, 593

Maitra, C., Paul, B., Haberl, F., \& Vasilopoulos, G. 2018, MNRAS, 480, L136

Masetti, N., D’Avanzo, P., Blagorodnova, N., \& Palazzi, E. 2014, ATel, 5999, 1

Meszaros, P., \& Nagel, W. 1985, ApJ, 298, 147

Mihara, T. 1995, PhD Thesis, University of Tokyo, Japan

Mitsuda, K., Inoue, H., Koyama, K., et al. 1984, PASJ, 36, 741

Müller, S., Ferrigno, C., Kühnel, M., et al. 2013, A\&A, 551, A6

Mushtukov, A. A., Suleimanov, V. F., Tsygankov, S. S., \& Poutanen, J. 2015, MNRAS, 447, 1847

Nishimura, O. 2014, ApJ, 781, 30

Nishiuchi, M., Koyama, K., Maeda, Y., et al. 1999, ApJ, 517, 436

Palmeri, P., Mendoza, C., Kallman, T. R., et al. 2003, A\&A, 410, 359

Paul, B., \& Naik, S. 2011, Bull. Astron. Soc. India, 39, 429

Postnov, K. A., Gornostaev, M. I., Klochkov, D., et al. 2015, MNRAS, 452, 1601

Pottschmidt, K., Kreykenbohm, I., Wilms, J., et al. 2005, ApJ, 634, L97

Protassov, R., van Dyk, D. A., Connors, A., et al. 2002, ApJ, 571, 545

Reig, P. 2011, Ap\&SS, 332, 1

Reig, P., \& Nespoli, E. 2013, A\&A, 551, A1

Sanna, A., Riggio, A., Burderi, L., et al. 2017, MNRAS, 469, 2

Schwarm, F. W., Ballhausen, R., Falkner, S., et al. 2017a, A\&A, 601, A99

Schwarm, F. W., Schönherr, G., Falkner, S., et al. 2017b, A\&A, 597, A3

Schwarzenberg-Czerny, A. 1989, MNRAS, 241, 153

Smirnov, N. V. 1939, Bull. Moscow Univ., 2, 3

Staubert, R., Trümper, J., Kendziorra, E., et al. 2019, A\&A, 622, A61

Strickman, M. S., Dermer, C. D., Grove, J. E., et al. 1996, ApJ, 464, L131

Tanaka, Y. 1986, in Radiation Hydrodynamics in Stars and Compact Objects, IAU Coll. 89, eds. D. Mihalas, \& K. A. Winkler (Berlin, Heidelberg: SpringerVerlag), Lect. Notes Phys., 255, 198

Trümper, J., Pietsch, W., Reppin, C., et al. 1978, ApJ, 219, L105

Tsygankov, S. S., Mushtukov, A. A., Suleimanov, V. F., et al. 2017, A\&A, 608, A17

Tsygankov, S. S., Doroshenko, V., Mushtukov, A. E. A., et al. 2019a, MNRAS, 487, L30

Tsygankov, S. S., Rouco, Escorial A., Suleimanov, V. F., et al. 2019b, MNRAS, 483, L144

Verner, D. A., Ferland, G. J., Korista, K. T., \& Yakovlev, D. G. 1996, ApJ, 465, 487

Wijnands, R., \& Wang, Q. D. 2002, ApJ, 568, L93

Wilms, J., Allen, A., \& McCray, R. 2000, ApJ, 542, 914

Woods, P. M., Kouveliotou, C., van Paradijs, J., et al. 1999, ApJ, 517, 431

Younes, G., Kouveliotou, C., Grefenstette, B. W., et al. 2015, ApJ, 804, 43

Zdziarski, A. A., Johnson, W. N., \& Magdziarz, P. 1996, MNRAS, 283, 193

Życki, P. T., Done, C., \& Smith, D. A. 1999, MNRAS, 309, 561 